\title{
Illuminating the role of caspases during Drosophila oogenesis
}

\author{
S Mazzalupo ${ }^{1,4}$ and L Cooley ${ }^{*, 1,2,3}$ \\ 1 Department of Genetics, Yale University School of Medicine, 333 Cedar Street, \\ New Haven, CT 06520-8005, USA \\ 2 Department of Cell Biology, Yale University School of Medicine, 333 Cedar \\ Street, New Haven, CT 06520-8005, USA \\ 3 Department of Molecular, Cellular \& Developmental Biology, Yale University, \\ New Haven, CT 05610, USA \\ ${ }^{4}$ Current address: Tucson Drosophila Stock Center, University of Arizona, \\ Tucson, AZ 85721-0088, USA \\ * Corresponding author: L Cooley, Department of Genetics, Yale University \\ School of Medicine, 333 Cedar Street, New Haven, CT 06520-8005, USA. \\ Tel: + 1203785 5067; Fax: + 1203785 6333; \\ E-mail: lynn.cooley@yale.edu
}

Received 08.8.05; revised 24.1.06; accepted 25.1.06; published online 10.3.06 Edited by E Baehrecke

\begin{abstract}
Cell death is a prominent feature of animal germline development. In Drosophila, the death of 15 nurse cells is linked to the development of each oocyte. In addition, females respond to poor environmental conditions by inducing egg chamber death prior to yolk uptake by the oocyte. To study these two forms of cell death, we analyzed caspase activity in the germline by expressing a transgene encoding a caspase cleavage site flanked by cyan fluorescent protein and yellow fluorescent protein. When expressed in ovaries undergoing starvation-induced apoptosis, this construct was an accurate reporter of caspase activity. However, dying nurse cells at the end of normal oogenesis showed no evidence of cytoplasmic caspase activity. Furthermore, although expression of the caspase inhibitors p35 or Drosophila inhibitor of apoptosis protein 1 blocked starvation-induced death, it did not affect normal nurse cell death or overall oogenesis in well-fed females. Our data suggest that caspases play no role in developmentally programmed nurse cell death.

Cell Death and Differentiation (2006) 13, 1950-1959.

doi:10.1038/sj.cdd.4401892; published online 10 March 2006
\end{abstract}

Keywords: oogenesis; nurse cell; caspase; apoptosis; germline; CFP; YFP; Drosophila melanogaster, phagocytosis; follicle cell

\begin{abstract}
Abbreviations: CFP, cyan fluorescent protein; Dcp-1, death caspase-1; Diap1, Drosophila inhibitor of apoptosis protein 1 (thread, FBgn 0003691); GFP, green fluorescent protein; YFP, yellow fluorescent protein
\end{abstract}

\section{Introduction}

Programmed cell death is a component of both invertebrate and vertebrate germline development (reviewed in Baum et al. ${ }^{1}$ and in Tilly ${ }^{2}$ ). In mammals there is a wave of oocyte cell death prior to or around birth, with continuing cell death during postnatal life. In the testis there is also perinatal and postnatal cell death presumably acting in conjunction with proliferation and differentiation to maintain the proper number of spermatazoa. In several examples from invertebrates, germ cells that have been acting as nurse cells to the developing oocyte undergo cell death once their function has been fulfilled. Hydra have nurse cells that undergo cell death before the growing oocyte phagocytoses them. Insect germline cell death has been studied in several species, including Ceratitis capitata, Dacus oleae, and most extensively in Drosophila melanogaster. ${ }^{1}$ The female germline of these insects is organized into egg chambers consisting of an oocyte and nurse cells that provide the growing oocyte with macromolecules, including proteins, mRNA, and organelles. During oogenesis, the nurse cells gradually transport maternal components to the oocyte, and then finally extrude all their remaining cytoplasmic contents into the oocyte, after which the nurse cell remnants die. ${ }^{3-5}$

The mechanism of nurse cell death in flies is unclear. Importantly, even though they are directly connected to the oocyte by intercellular bridges (ring canals) throughout most of oogenesis, the oocyte survives while the nurse cells die. Therefore, either the effectors of cell death must be completely sequestered within the nurse cells, or nurse cell death only occurs after the ring canal connections between the nurse cell remnants and oocyte are severed. A third possibility is that the oocyte is actively protected from cell death.

Dying nurse cells display some of the features of apoptotic cells. Apoptosis is a way of removing defective or unwanted cells without inducing a toxic inflammatory response in the surrounding tissue. Morphological changes such as cell blebbing, chromatin condensation, clumped organelles that are still structurally intact, and nuclear membrane convolution characterize apoptosis. Also, apoptotic cells have molecular hallmarks, most notably the activation of a cascade of proteases called caspases that cleave downstream targets, some of which lead to the morphological changes seen during apoptosis such as DNA laddering. ${ }^{6}$ Molecular assays such as terminal deoxyribonucleotidyl transferase (TdT)-mediated biotin-16-dUTP nick-end labelling (TUNEL) and Annexin V staining indicate dead cells, but not exclusively apoptotic cells. $^{7}$

However, nurse cell death is independent of known inducers of Drosophila somatic cell apoptosis. Females that lack apoptosis inducers (hid, reaper, grim) in the germline produce egg chambers with nurse cell remnants that are TUNEL positive, the females are fertile, and they produce normal eggs. ${ }^{4}$ Interestingly, germ cell death in Caenorhabditis elegans is also independent of the signals that trigger somatic cell apoptosis. In somatic cells, apoptosis is dependent on a BH3-containing protein called EGL-1 that promotes activation of the caspase CED-3. However, apoptosis of the germline cells still occurs in these egl-1 mutants. ${ }^{8}$ These results 
suggest that germ cell death is regulated by a germlinespecific mechanism.

Since nurse cell death is independent of known apoptosis inducers, it is important to determine whether their death is apoptotic. Initial investigations suggested that it was. TUNELpositive nurse cell nuclei, ${ }^{4}$ DNA laddering, and condensation of chromatin as seen by electron microscopy ${ }^{3}$ were noticed in late-stage nurse cell remnants. Neighboring cells or professional phagocytes engulf apoptotic blebs, to remove the dying cells. ${ }^{9,10}$ One electron microscopy investigation documents follicle cells engulfing stage 13 nurse cell fragments. ${ }^{5}$ When an effector caspase (death caspase-1 (dcp-1), FBgn 0010501) mutant was found, ${ }^{11}$ germline clones lacking $d c p-1$ were unable to complete nurse cell cytoplasm transport, suggesting caspase involvement in nurse cell function.

Evidence is now accumulating for nonapoptotic cell death in late-stage nurse cells. When Drosophila inhibitor of apoptosis protein 1 (Diap1) is ectopically expressed in the ovary, nurse cells still undergo cell death. ${ }^{12}$ Flies lacking Dredd (an initiator caspase) only have an immunological defect. ${ }^{13}$ Flies lacking Dronc (another initiator caspase) are homozygous lethal. ${ }^{14-16}$ However, germline clones of dronc do result in embryos, and no oogenesis defect was reported. ${ }^{16}$ Finally, the mutations reported in $d c p-1$ were later found to affect two genes. ${ }^{17}$ When $d c p-1$ alone is mutated, there is no effect on late-stage nurse cell death. ${ }^{12,18}$ Therefore, genetic analysis of individual caspase-encoding genes has not provided evidence for caspase function during normal oogenesis.

Immature Drosophila egg chambers also undergo sporadic cell death before the onset of yolk accumulation (vitellogenesis) by the oocyte. This mid-stage egg chamber death can be stimulated by incubation in etoposides or by adverse environmental conditions such as overcrowding or lack of food. ${ }^{19}$ The pre-vitellogenic stage of egg chamber development appears to be a key checkpoint for general health, as many mutations that disrupt signaling or metabolism result in stage $8 / 9$ arrest. $^{19,20}$ In addition, ovaries from starved females have degenerating egg chambers in region 2 of the germarium, ${ }^{19}$ which contains nascent egg chambers. Thus, females can eliminate egg chambers either before they are fully assembled (region 2) or before they are endowed with yolk (stage 8/9). Cell death in both these stages displays the hallmarks of apoptosis such as TUNEL-positive nuclei, cell membrane blebbing, and chromatin condensation. 5,12,19,21

One complicating issue with regard to the examination of apoptosis is the presence of multiple caspase genes. In Drosophila, there are several caspase genes and thus redundancy could be masking their involvement in oogenesis. To resolve the role of apoptosis in nurse cells, we developed transgenic Drosophila lines to analyze caspase activity in egg chambers. We made a detector of caspase activity using a caspase cleavage site flanked by fluorescent proteins. In ovaries from starved females, the reporter showed evidence of caspase activity in degenerating, pre-vitellogenic egg chambers. Starvation-induced egg chamber death and caspase activation was inhibited by overexpression of the caspase inhibitor p35 or Diap1. In contrast, nurse cells from well-fed females showed no evidence of caspase activation, even after the completion of cytoplasm transport to the oocyte late in oogenesis. In addition, caspase inhibitors had no effect on normal oogenesis.

Our evidence, and recently published caspase mutant data, ${ }^{14-16}$ do not support a role for caspase-dependent apoptosis during or after cytoplasm transport in egg chambers, and suggest that apoptosis is not necessary for the normal germline development during oogenesis.

\section{Results}

\section{Detection of in vivo caspase activity}

To study apoptosis during oogenesis, we developed the CIETDY reporter to detect caspase activity in living ovaries. There are seven known caspases in the Drosophila genome. dronc (FBgn 0026404), dredd (FBgn 0020381) and sometimes dream (FBgn 0033051) are reported as the apical (or initiator) caspases based on the large prodomains evident in their protein sequences. These are the upstream caspases that cleave and activate the effector, or downstream, caspases (Dcp-1, Drice, Damm, and Decay). ${ }^{6}$ Studies using commercially available caspase substrates found that Dronc $^{22}$ and Dredd ${ }^{23}$ could both cleave lle-Glu-Thr-Asp (IETD) after the aspartate residue. We made a reporter containing an IETD site to detect apical caspase activity in germline cells. Using a strategy similar to the DEVD-containing constructs used by Luo et al., ${ }^{24}$ we made a construct consisting of cyan fluorescent protein (CFP) and yellow fluorescent protein (YFP) linked by the amino-acid sequence Val-lle-Glu-Thr-Asp-Gly-Ala (VIETDGA) (Figure 1a). A mitochondrial outer membrane targeting sequence ${ }^{17}$ was fused to the C-terminus of YFP. Upon cleavage by caspases, the CFP would no longer be tethered to the mitochondrion-
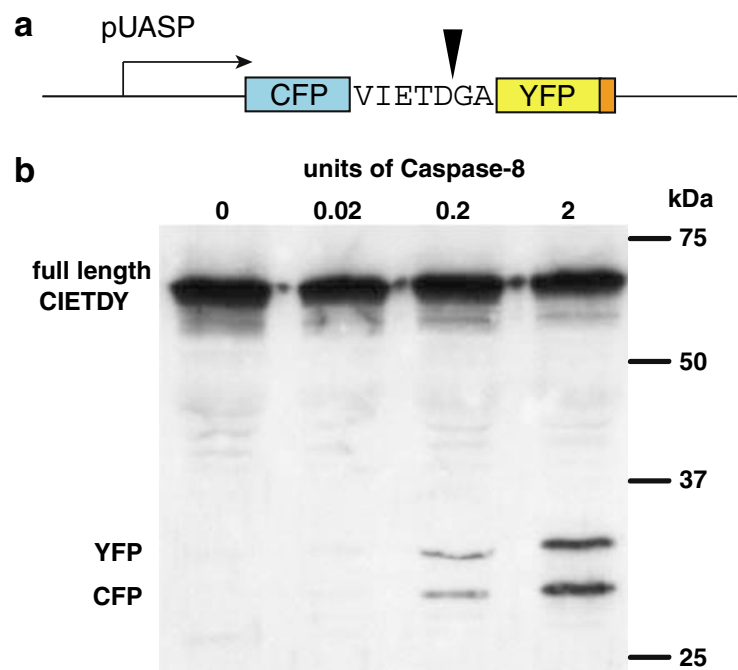

Figure 1 Construction of a caspase detecting transgene. CFP and YFP were linked by the amino-acid sequence VIETDGA, which contains the IETD cleavage site recognized by Dronc and Dredd caspases. The C-terminal of YFP was fused to a mitochondrial outer membrane targetting sequence. (a) The final construct as it was ligated into UASp before injection. The arrowhead indicates the site of caspase cleavage. (b) The verification of caspase cleavage of the CIETDY construct was carried out on ovary extracts using purified human caspase-8. Western blot analysis was performed with an antibody against GFP that crossreacts with YFP and CFP, and results are shown for 0-2 U of caspase-8 
localized YFP and could diffuse throughout the cell. The YFP emission of $\sim 530 \mathrm{~nm}$ (mitochondrial) would be spatially distinct from the CFP emission of $\sim 480 \mathrm{~nm}$ (cytoplasm). This CFP-linker-YFP construct (hereafter referred to as CIETDY, pronounced 'see - it - die') was cloned into UASp ${ }^{25}$ to take advantage of the UAS-Gal4 expression system. Independent germline transformants were obtained on each chromosome, and flies were crossed to Gal4 lines to obtain germline expression. All results reported in this paper are from flies expressing CIETDY exclusively in the germline. Western analysis of protein extracts from ovaries showed that CIETDY was expressed equivalently in all lines (data not shown). P[CIETDY-2]; nanos-Gal4VP16 (nosCIETDY-2) and P[CIETDY-3], nanos-Gal4VP16/TM3 (nosCIETDY-3) ovaries were chosen for further analyses. We incubated protein extracts from these ovaries with purified human caspase-8, which preferentially cleaves after IETD. ${ }^{6}$ Western analysis revealed that CIETDY was cleaved, and the amount of cleavage products present was dependent upon caspase-8 concentration (Figure 1b). However, purified human caspase-3 (a downstream or effector) caspase was also tested with the CIETDY-containing protein extracts, and it does not cleave the CIEDTY (data not shown).

All nosCIETDY lines showed an identical expression pattern in healthy egg chambers. The CFP and YFP fluorescence patterns were mitochondrial, and in live egg chambers the signals moved as mitochondria did $^{26}$ (data not shown). In nosCIETDY egg chambers, the CFP and YFP signals overlapped completely (Figures $2 \mathrm{a}$, lower egg chamber, and $b$, white color) when there was no evidence of apoptosis. As expected from this germline driver, the signal was wholly in the germline cells.

To test for caspase activity detection, we deprived nosCIETDY females of an amino-acid source by growing them on grape juice plates ('starved'). This treatment leads to ovaries that are composed mostly of normal pre-vitellogenic egg chambers, with one to three apoptotic egg chambers per ovary, always located next to the oviduct (Figure $3 \mathrm{~b}$ ). The starved nosCIETDY females contained dying egg chambers that showed evidence of caspase activity, with the CFP and YFP fluorescence no longer completely co-localized (Figure 2a, upper egg chamber). The separation of CFP from YFP became more pronounced as apoptosis proceeded (Figure 2 and Supplementary information - Supplementary Figure S1). The CFP and YFP signals in healthy egg chambers completely overlapped (Figure $2 b$ ), while at the beginning of the degeneration process, the overall 'texture' of the fluorescence signal changed, with the YFP signal clumped and the CFP signal diffuse in the cytoplasm (Figure 2c). In late-apoptotic egg chambers (Figure 2d), cytoplasmic remnants had either diffuse CFP fluorescence or clumped YFP fluorescence, an expected result if caspases were cleaving the CIETDY. This very striking difference in CFP and YFP localization was evident in both fixed and live ovaries; however, for all images in this paper, fixed tissue was used.

In the degenerating egg chambers, the apoptotic blebs of the dying germline cells seemed to be phagocytosed by the follicle cells. The follicle cells are somatic epithelial tissue surrounding the 16 germline cells. We immunostained with the 1B1 antibody to hu-li tai shao or used phalloidin staining in

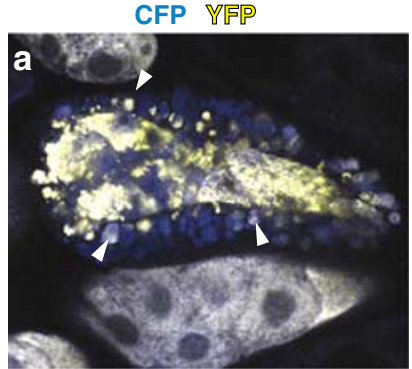

$1 B 1$ CFP YFP

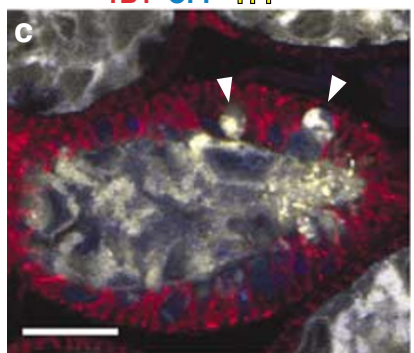

CFP YFP
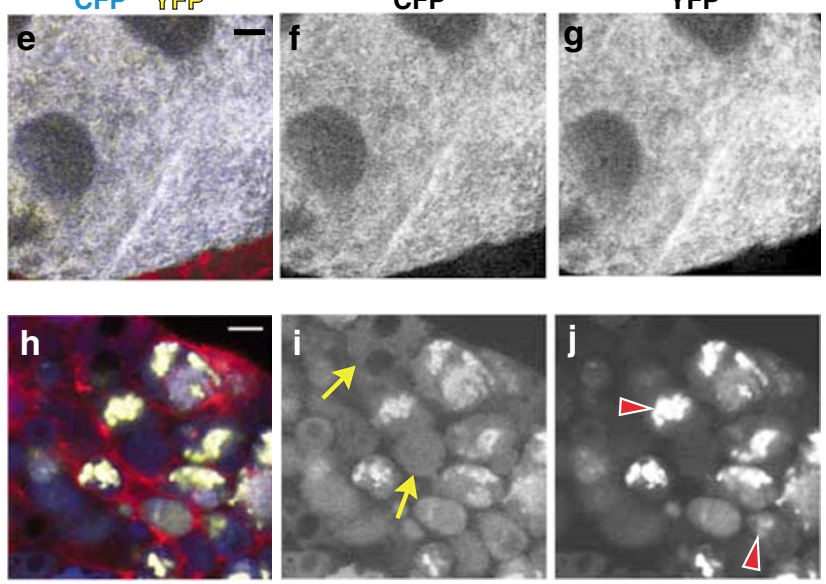

Figure 2 Detection of caspase activity in starvation-induced apoptosis of egg chambers. (a) Fluorescence micrograph of egg chambers from a starved female expressing the CIETDY construct. The upper egg chamber is degenerating, while the lower one remains healthy. The arrowheads indicate blebs of germline cytoplasm. (b) A healthy egg chamber with CFP and YFP completely colocalized. Red 1B1 antibody staining highlights the follicle cell epithelial layer that surrounds the germline cells. (c) An egg chamber in the early stages of apoptosis in which there are blebs in the follicle cells and some separation of CFP and YFP fluorescence. Arrowheads indicate blebs of germ cell cytoplasm being engulfed by surrounding follicle cells. (d) A late-apoptotic egg chamber stained with rhodamine-conjugated phalloidin (red) to reveal membrane-associated F-actin. (e-g) A magnification of the boxed portion of the egg chamber in (b) shown as $1 B 1$ staining, CFP and YFP merged (e) and then separated into CFP only (f) and YFP only ( $\mathbf{g})$ images. $(\mathbf{h}-\mathbf{j})$ A magnification of the boxed portion of the egg chamber in (d) shown as F-actin staining, CFP and YFP merged (h) and then separated into CFP only (i) and YFP only (j). The red arrowheads indicate very bright YFP that is likely to be associated with mitocondria. The yellow arrows point to CFP-positive, YFP-negative blebs. The areas with no fluorescence signal in (i) are yolk or nuclear fragments in the blebs that exclude the cytoplasmic CFP. (b-d) Bar: $25 \mu \mathrm{m}$. (e, h) Bar: $10 \mu \mathrm{m}$

order to indicate follicle cell membranes. Follicle cells of healthy egg chambers were in an even row around the germline cells with no CIETDY in the somatic follicle cell layer (Figure 2b). In early apoptotic egg chambers, blebs of fluorescent germline cytoplasm were present that protruded into the follicle cell layer (Figure $2 \mathrm{a}$ and c, arrowheads). In the 

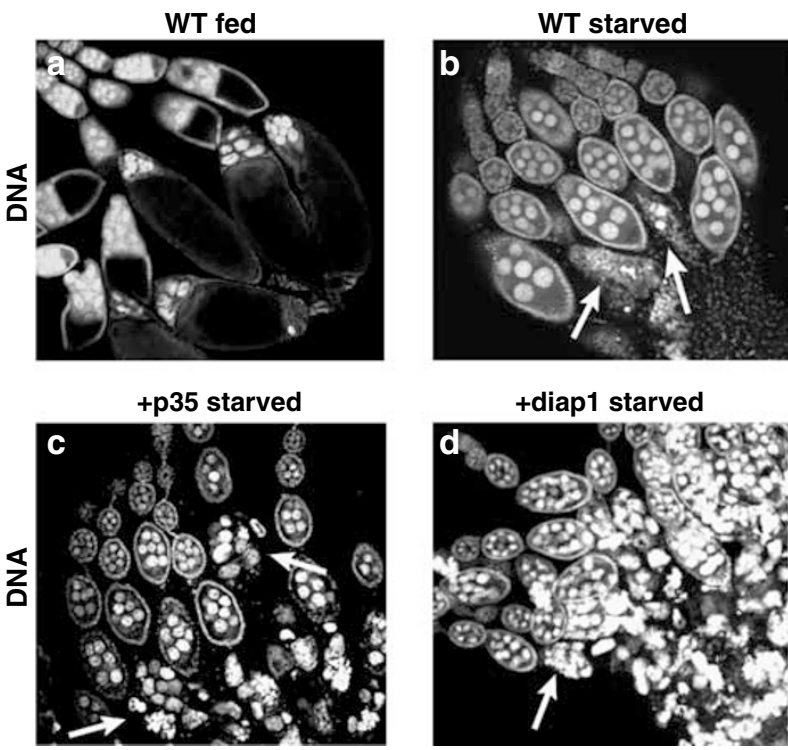

Figure 3 Effect of inhibiting caspases in starved egg chambers. Ovaries were stained to indicate DNA, and micrographs were taken with a $\times 10$ objective in order to show the range of stages in the ovary. (a) All stages of egg chambers are present in ovaries from fed wild-type females. (b-d) Egg chambers from starved females. (b) Ovaries from wild-type females contain egg chambers only through stage 8, after which they degenerate (arrows). Note the condensed chromatin and absence of nuclear structure in degenerating egg chambers. Starved females expressing p35 (c) or Diap1 (d) have undead germline tissue that accumulates at the base of the ovary (arrows in $\mathbf{c}$ and $\mathbf{d}$ ). The undead egg chambers have uncondensed germline chromatin and no follicle cell nucle

late apoptotic egg chambers, the germline was wholly encompassed in blebs that were inside the follicle cells, and often these blebs contained what appeared to be mitochondrial fragments (Figure $2 \mathrm{~d}$ ). These results are consistent with electron microscopy studies of mid-stage degenerating egg chambers $^{21}$ in which the healthy follicle cells engulf blebs of cytoplasm from the nurse cells and the oocyte, and the blebs sometimes contain organelles, yolk particles and nuclear material.

The separation of CFP and YFP in apoptotic cells had a characteristic pattern. In contrast to healthy cells in which CFP and YFP co-localized in a mitochondrial pattern (Figure $2 \mathrm{e}-\mathrm{g}$ ), apoptotic blebs of germline tissue had distinct patches of diffuse CFP with little or no YFP (Figure $2 \mathrm{~h}-\mathrm{j}$, arrows), or clumps of bright YFP with some CFP fluorescence (Figure $2 \mathrm{~h}-\mathrm{j}$, arrowheads). Some blebs had patches of equally intense YFP and CFP indicating uncleaved CIETDY. The CFP-only patches likely represent CFP in the cytoplasm liberated by CIETDY cleavage, while the bright YFP patches are probably aggregated mitochondria.

\section{Blocking apoptosis in the germline}

Another approach to studying apoptosis during oogenesis is to block caspase activation. Germline expression of Diap1 can block starvation-induced apoptosis of the germline in previtellogenic egg chambers. ${ }^{12}$ We tested whether expression of p35, a baculovirus-derived caspase inhibitor, had a similar ability. We used nos-Gal4 to drive expression of either $\mathrm{p} 35^{27}$ or Diap $1^{12}$ in the germline of females. Wild-type flies that were fed normally had egg chambers of all stages (Figure 3a) while starved ovaries from wild-type flies had no egg chambers beyond stages 7-8 (Figure $3 \mathrm{~b}$ ). We used a DNA-binding dye to visualize nuclei, and follicle cell nuclei surrounded these degenerating egg chambers (Figure 3b, arrows). The addition of p35 or Diap1 expression in the germline caused a dramatic change in the morphology of ovaries from starved females. The base of these ovaries was filled with a mass of unhealthy, undead egg chamber tissue, including large uncondensed nurse cell nuclei (Figure $3 c$ and $d$ ). The residual egg chambers were devoid of follicle cell nuclei, giving them a 'bald' appearance (Figure $3 c$ and $d$, arrows). We immunostained with 1B1 to indicate follicle cell membranes, and showed that residual anucleate follicle cell tissue surrounded the germline cells, but membranes no longer appeared regular (Figure $4 \mathrm{a}$ and b). Thus, the germline was substantially protected from starvation-induced apoptosis by germline expression of caspase inhibitors. Conversely, the follicle cells were not protected and presumably proceeded with their normal role in egg chamber degeneration before losing their nuclei.

We tested whether caspase inhibitors affected cleavage of the CIETDY reporter by combining germline expression of nosCIETDY with either p35 or Diap1 and culturing females in starvation conditions. CIETDY was uncleaved in the healthy egg chambers surrounded by follicle cells (Figure 4f, asterisks). In the egg chambers with undead germline cells that had lost their follicle cells (Figure 4f, arrows), the CIETDY did appear in CFP- or YFP-positive patches. However, the patches were larger than the $5-10 \mu \mathrm{m}$ blebs seen in Figure 2. Closer examination of 'bald' egg chambers (Figure $4 \mathrm{~g}$ ) showed that the CFP and YFP signals mostly co-localized, but were in condensed fragments not associated with nuclei (Figure 4gi and ii). A few smaller fragments could be seen that were either CFP-only (Figure 4gi, black arrowhead) or YFPbright (Figure 4gi, white arrowhead). These could be the products of proteolytic activity or caspase activity that escaped inhibition.

Careful examination of ovaries from starved females expressing either caspase inhibitor revealed an interesting intermediate stage of egg chambers. Occasionally egg chambers with an uneven follicle cell epithelium were present at the interface between healthy pre-vitellogenic egg chambers and the bald egg chambers with uncondensed nurse cell nuclei. These egg chambers had blebs of germline tissue in the process of being phagocytosed by the follicle cells (Figure 4c, arrowhead), even though the nurse cells appeared healthy with large nuclei and uncleaved CIETDY (Figures 4c and $d$ ). This suggested that follicle cells responded to the starvation-induced signal for egg chamber apoptosis and began to phagocytose the nondegenerating germline. An occasional apoptotic follicle cell nuclei was seen (Figure $4 d$, arrowhead) suggesting that the follicle cells still undergo apoptosis.

\section{No detection of caspase activity in normal development of late-stage nurse cells}

The results in Figures 2, 3, and 4 convinced us that we could detect caspase activity in the germline with the CIETDY 
reporter, and that we could inhibit environmentally induced apoptosis by expressing p35 or Diap1 in the germline. We used CIETDY to examine late-stage egg chambers for evidence of caspase activation, where developmentally regulated nurse cell death was occurring. We examined ovaries from females fed on wet yeast paste for $36 \mathrm{~h}$ to get an optimal number of stage 10-14 egg chambers. Several
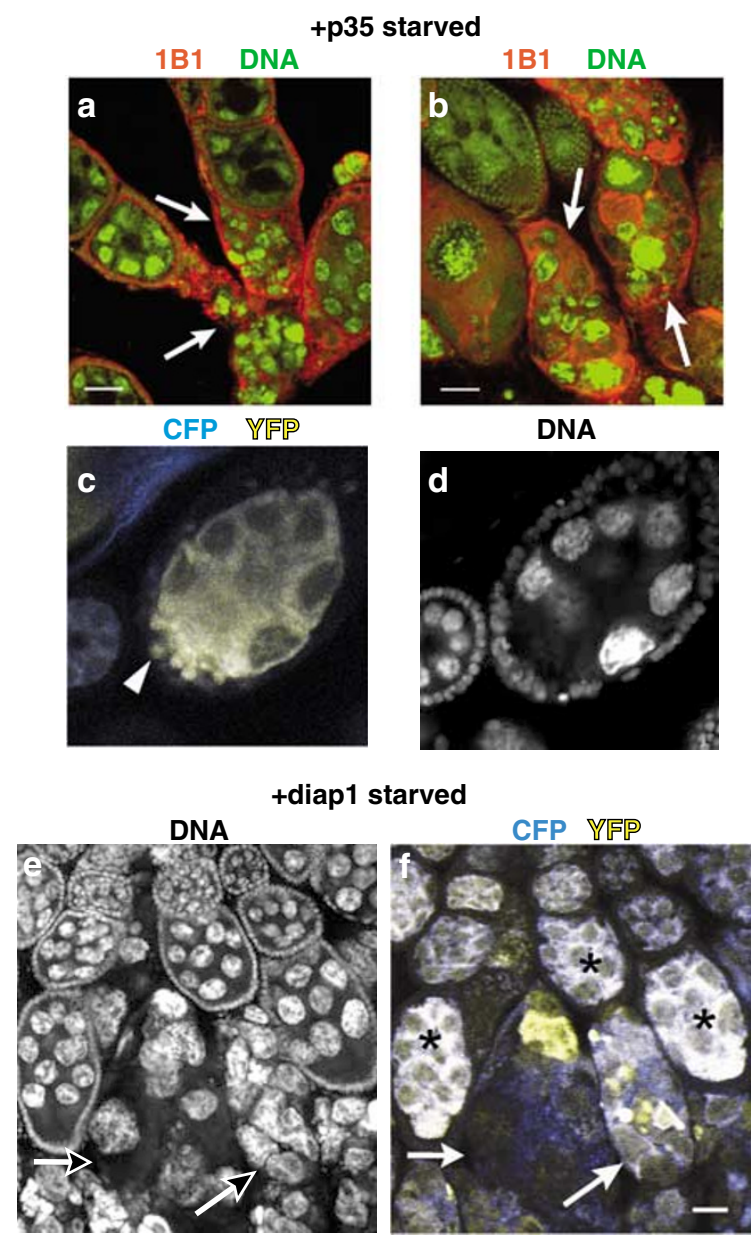

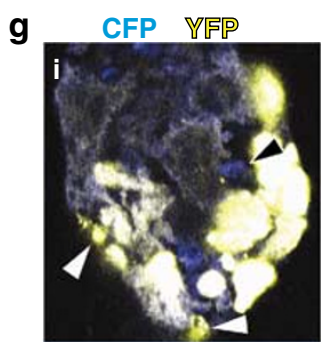

CFP

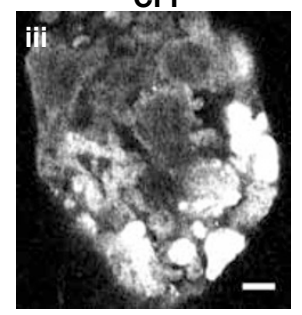

DNA

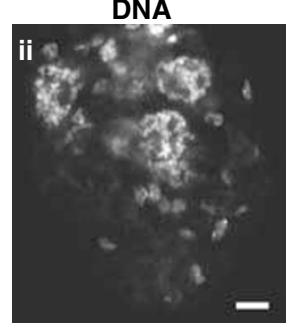

YFP

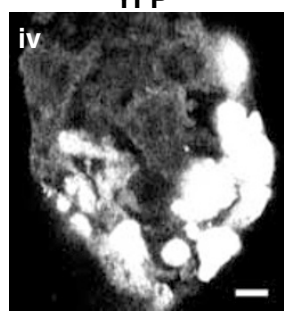

CIETDY lines were examined (PICIETDY-X], P[CIETDY-2], or $P[C I E T D Y-3])$ with many germline Gal4 drivers ( $P[$ nanosGal4VP16], P[tubulin-Gal4] or P[COG-Gal4VP16; NGT40; nanos-Gal4VP16]). In addition, we examined 16 movies of live egg chambers (data not shown). In all images of fixed (as represented in Figure $5 \mathrm{a}-\mathrm{h}$ ) and live egg chambers, no separation of CFP and YFP fluorescence was seen in nurse cells prior to or during rapid cytoplasm transport (stages 10 and 11). CFP and YFP always co-localized, leading us to conclude that caspase activity does not precede the developmentally regulated nurse cell death seen after stage 10 (Figure 5a). As the nurse cell remnants began disappearing from the egg chambers (stages 11 and 12), we still saw no evidence of caspase activity (Figure $5 b$ ). By stage 14, there is no CIETDY signal remaining outside the oocyte. (Figure $5 \mathrm{c}$ and d). Between stages 10 and 14, the follicle cells did not phagocytose CIETDY from the nurse cells, and we saw none of the conspicuous blebs present in the degenerating stage 7-8 egg chambers.

We looked carefully at stage 13 egg chambers because of reports in the literature that nurse cell nuclei become TUNELpositive $^{3-5}$ at this stage (Figure $5 e-h$ ). During stage 13 , the CIETDY reporter behaved differently; the fluorescent proteins became localized within nurse cell nuclei rather than remaining in the cytoplasm, suggesting that nucleo-cytoplasmic trafficking is altered or disrupted during stage 13. Within these nuclei, CFP and YFP fluorescence was always evenly distributed and there were no apoptotic blebs visible. The intensity of CFP and YFP fluorescence varied within nuclei; some nuclei had only CFP fluorescence and others had bright YFP fluorescence in addition to CFP (Figure 5e-h), somewhat reminiscent of what we observed in the apoptotic blebs of degenerating germline cells (Figure $2 \mathrm{~h}-\mathrm{j}$ ). The behavior of CIETDY during stage 13 could possibly be due to caspase activation and cleavage of the reporter. However, it is not clear where such a cleavage might have taken place since the reporter proteins were localized to an unexpected compartment. Was the CIETDY cleaved in the cytoplasm and the cleavage products became localized to nuclei, or did cleavage

Figure 4 Examination of starved egg chambers expressing caspase inhibitors in the germline. (a, b) Egg chambers from starved females expressing p35 were stained for DNA (YO-PRO, green) and follicle cell membranes (1B1, red). Follicle cell nuclear staining is no longer present in the posterior undead egg chambers (arrows). Although follicle cell nuclei are gone, 1B1 staining shows that follicle cell membranes are still present. An egg chamber from a starved female expressing p35 and nosCIETDY in the germline showing either DNA (d) or CFP and YFP (c) fluorescence. Although the germline cells look healthy with no separation of CFP and YFP, the follicle cells are attempting engulf germline blebs (arrowhead in $\mathbf{c}$ ). Germline nuclei have diffuse staining, while some of the follicle cell nuclei are starting to condense (arrowhead in d) as if undergoing apoptosis. (e-g) Egg chambers from starved females expressing Diap1 and nosCIETDY in the germline showing DNA or CFP and YFP fluorescence. 'Bald' egg chambers (arrows in $\mathbf{e}$ and $\mathbf{f}$ ) have lost their surrounding follicle cell nuclei, but contain large uncondensed germline nuclei. Healthy egg chambers (asterisks) still have their follicle cell nuclei. Closer examination of a single 'bald' egg chamber (g) showed that CFP and YFP fluorescence were largely co-localized in large patches removed from germline nuclei. In some examples, CFP and YFP show intensity differences. Some patches have more CFP than YFP fluorescence (black arrowhead) and some have these intensities reversed (white arrowheads). (a-f) Bar: $25 \mu \mathrm{m}$. (g) Bar: $10 \mu \mathrm{m}$ 

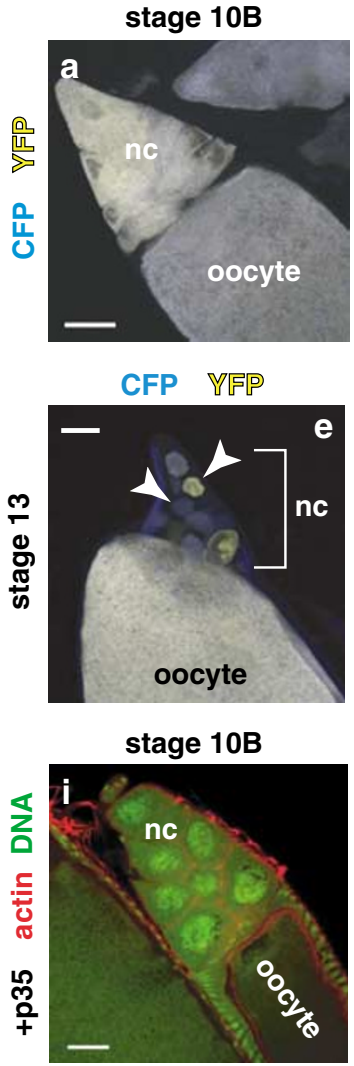

stage 10B

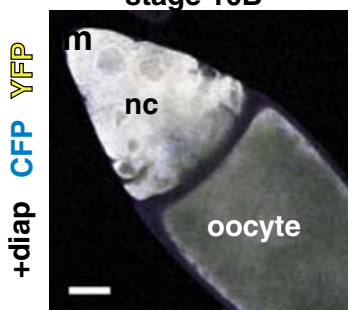

stage 12

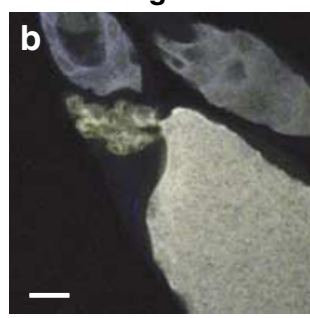

DIC

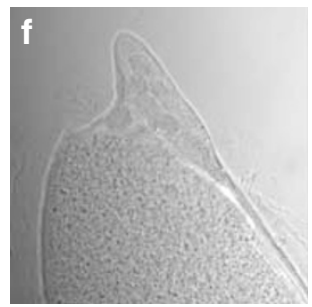

stage 13

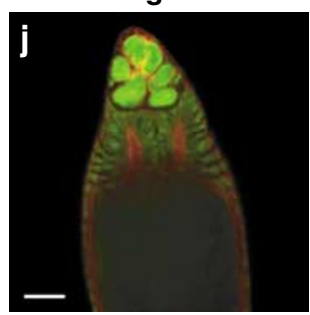

stage 13

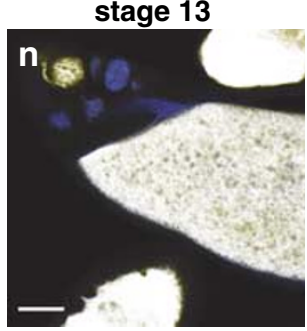

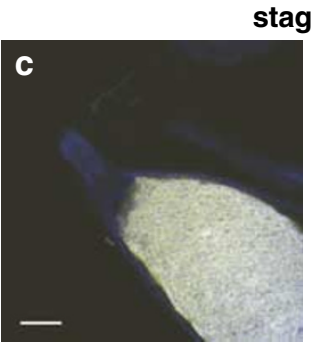

YFP
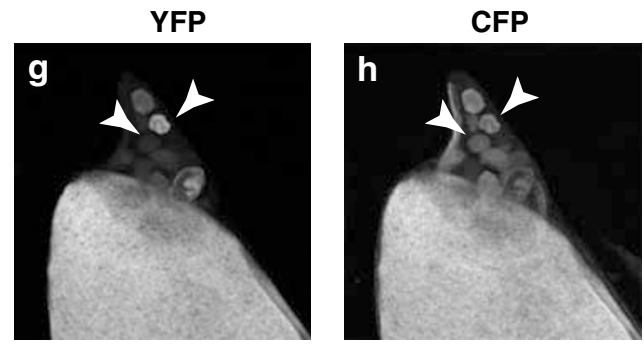

stage 14
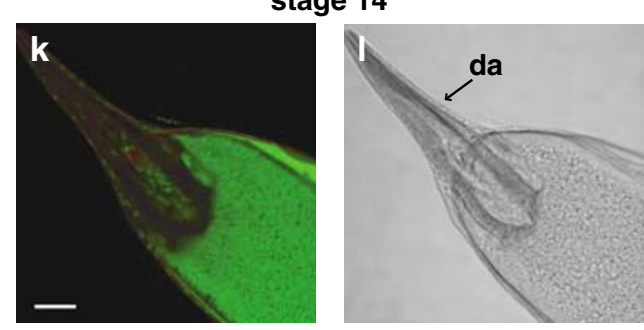

stage 14
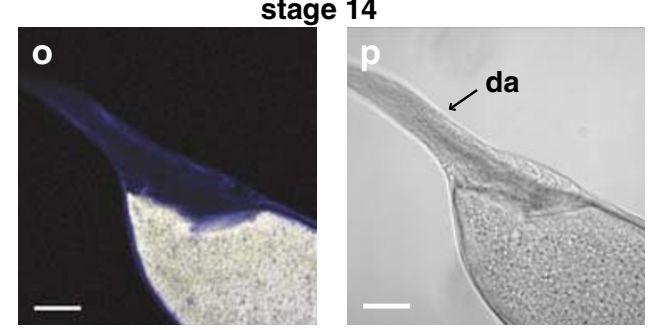

Figure 5 Late-stage egg chambers expressing p35 or Diap1 in the germline are unaffected during nurse cell death and oocyte development. (a-d) Confocal and DIC images of stage 10-14 egg chambers from well-fed females expressing CIETDY in the germline. No separation of the CFP and YFP signals was seen. (e-h) A stage 13 egg chamber that has the absence (or reduction) of one fluorophore in some nurse cell remnants (arrowheads). (i-I) Confocal and DIC images of stage 10-14 egg chambers from well-fed females expressing p35 in the germline. DNA is stained with YO-PRO-1 (green) and F-actin with rhodamine phalloidin (red). ( $\mathbf{m - p}$ ) Confocal and DIC images of egg chambers from well-fed females expressing germline diap1 and CIETDY. In stage 14 egg chambers, the signal that is apparent at the CFP emission wavelength is the autofluorescence of the dorsal appendages. $\mathrm{nc}=$ nurse cell remnants, $\mathrm{St} .=$ stage, da $=$ dorsal appendages. Bar: $50 \mu \mathrm{m}$

occur within nuclei? An alternative possibility is that other proteolytic enzymes activated in stage 13 nurse cell remnants were randomly cleaving the CIETDY. Surviving CFP or YFP moieties would be responsible for the fluorescence pattern we observed in stage 13 nurse cell remnants. Further investigation of this interesting phenomenon is beyond the scope of this paper.

\section{Expression of caspase inhibitors in the late-stage egg chambers}

The lack of detectable caspase activity in stage 10-12 egg chambers was consistent with the conclusion that caspases are not necessary for nurse cell death. Moreover, Peterson et al. ${ }^{12}$ reported that expression of Diap1 in the ovary did not affect nurse cell death. To extend these studies, we examined nurse cell behavior in egg chambers expressing either p35 or Diap1 along with CIETDY (Figure 5). After fixation and staining for DNA and F-actin (Figure 5i-l) or for CIETDY (Figure $5 m-p$ ), these ovaries were imaged using DIC and confocal microscopy and examined for any phenotypes. The egg chambers expressing Diap1 and p35 showed no differences in the timing or the appearance of rapid cytoplasm transport. Nurse cell cytoplasmic actin bundles formed during stage 10B, rapid cytoplasm transport occurred normally, and the females were fully fertile. As with wild-type egg chambers, there was no separation of CFP from YFP prior to or during rapid cytoplasm transport. In addition, after examining over 30 egg chambers for each genotype, nuclear staining with either 
TO-PRO-1 or YO-PRO-1 indicated that the DNA was identical in appearance to wild-type egg chambers at all timepoints. However, we did not perform TUNEL on the egg chambers, and thus we cannot comment upon similarities or differences in DNA fragmentation between wild-type and p35- or Diap1expressing egg chambers.

\section{Reduction of dronc caspase}

To investigate the effect of inhibiting apical caspase function, we created a transgene to carry out RNA-interference of the caspase dronc. The transgene encodes hairpin RNA to produce a double-stranded RNA that contains base pairs $265-765$ of the coding region of dronc. We tested the ability of this transgene to rescue reaper-induced apoptosis in the eye (see Supplementary information - Supplementary Figure S2), and found that expression of one copy of the dronc-RNAi transgene rescued reaper-induced cell death in the eye moderately well. However, expressing the dronc RNAi construct with a strong germline driver in starved ovaries did not prevent egg chamber apoptosis (data not shown). Furthermore, semi-quantitative RT-PCR of ovarian RNA failed to show a reduction in dronc mRNA levels (data not shown). Thus, attempts to reduce dronc in the germline were unsuccessful and the RNAi approach could not be used to examine the effects of caspase loss on normal nurse cell death. Indeed, the lack of published reports using RNAi in the Drosophila germline suggests that this approach may not work efficiently in germline cells.

\section{Discussion}

The successful production of every egg in Drosophila requires the support and then death of 15 nurse cells. Despite being directly connected by large cytoplasmic bridges, the oocyte survives the death of its sister cells. The mechanisms for carrying out developmentally regulated nurse cell death while not affecting the oocyte are of critical importance to oocyte development. Early cellular and genetic information suggested that nurse cells die by apoptosis, and that caspase actvity is needed for the final transfer of cytoplasm into the maturing oocyte. However, our data strongly suggest that nurse cell cytoplasm transport and subsequent death are independent of caspase function. Therefore, other mechanisms need to be explored for developmentally regulated cell death.

\section{Absence of caspase activity during normal germline development}

We showed by several means that caspases are not needed for normal oogenesis. The CIETDY caspase activity detector accurately reported apoptosis during egg chamber death induced by starvation; however, the construct showed no evidence of apical caspase activation in the cytoplasm before or during normal nurse cell death. The expression of two caspase inhibitors, p35 and Diap1, had no effect on nurse cell death despite significantly blocking starvation-induced egg chamber apoptosis. This is in agreement with previous work showing no effect on nurse cells caused by the overexpression of Diap $1,{ }^{12}$ the loss of several individual caspase ${ }^{1}$ or the loss of apoptosis activators. ${ }^{4}$ Taken together, the CIETDY and caspase inhibitor data provide a compelling argument that developmentally regulated nurse cell death is not caspasedependent apoptosis and that caspases are not necessary for female germline development.

The one possible exception to this conclusion is stage 13, during which we observed nurse cell nuclei with CFP and no YFP. This could indicate that CIETDY was cleaved during stage 13 and that caspases are involved with the removal of nurse cell remnants from egg chambers. However, the presence of CFP and YFP in nuclei at this stage was unexpected and difficult to interpret; the reporter was designed to show separation of the two fluorescent proteins within the cytoplasm of cells upon caspase cleavage. These results suggest that it would be necessary to use a reporter for caspase activity that was not dependent upon subcellular localization in order to investigate the presence of stage 13 caspase activity. However, whether CFP becomes separated from YFP by the action of caspases or other proteases and why they end up in the nuclei are intriguing questions that require additional research.

\section{Mechanism of nurse cell death}

The mechanisms to trigger and carry out nurse cell death remain elusive. Researchers have described many cell death alternatives to apoptosis, including caspase-independent cell death, ${ }^{28}$ paraptosis, ${ }^{29}$ necroptosis, ${ }^{30}$ autophagy and necrosis. Only the latter two mechanisms of cell death have been well characterized (reviewed in Edinger and Thompson ${ }^{31}$ ). Autophagy is a process in which a membrane encircles a volume of cytoplasm to form an autophagic vacuole. Then, these vacuoles dock and fuse to lysosomes to begin the digestion of their contents. Necrosis is the form of cell death that occurs in response to cellular injury. It is the form of cell death observed when cells lack ATP, characterized by the rupture of organelles and the plasma membrane.

Fly lines now exist to study autophagy, ${ }^{32}$ but the cell death of late-stage nurse cells does not bear a resemblance to autophagy. We observed no evidence of autophagic vesicles in late-stage egg chambers expressing either the Atg5-GFP or LC3-GFP fusion proteins (S Mazzalupo and L Cooley, unpublished). Necrosis cannot be discounted easily because Annexin $\mathrm{V}$ staining ${ }^{33}$ and $\mathrm{TUNEL}^{34}$ can be observed in necrotic cells. More research focusing on the proteases involved ${ }^{35}$ might allow a molecular test for necrosis in nurse cells.

\section{Phagocytosis by follicle cells}

The engulfment of germline material by follicle cells is an intriguing finding. Follicle cells are not professional phagocytes, but in the apoptotic egg chambers they are the cells engulfing the nurse cell and oocyte blebs. ${ }^{21}$ Moreover, even in the p35- or Diap1-expressing egg chambers, the follicle cells tried engulfing germline cytoplasm before they became anucleate. Research into phagocytosis has focused on both recognition and engulfment. In C. elegans, the ced-1/-6/-7 and 
ced-2/-5/-10/-12 pathways have been found to be partially redundant for phagocytosis during cell death in nematodes. ${ }^{9}$ Several Drosophila homologues for these genes are known, ${ }^{17}$ but most of the published research examines professional phagocytes. Follicle cells functioning as phagocytes would be expected to express one or more recognition receptors. Recognition receptors for hemocytes/macrophages include Croquemort (crq, FBgn 0015924), Scavenger receptor class C, type I (Sr-Cl, FBgn 0014033), and Peptidoglycan recognition protein LC ( $p G R P-L C, F B g n$ 0035976). In the example of mammary gland involution, vertebrate epithelial cells of the mammary gland express CD36 when they engulf apoptotic neighbors during involution. ${ }^{36}$ We found that the CD36-related protein Crq was not expressed on follicle cells during the engulfment of apoptotic blebs (S Mazzalupo and L Cooley, unpublished). Draper (drpr, FBgn0027594), a Drosophila ortholog of CED-1 that is expressed on hemocytes/macrophages, ${ }^{17}$ is another molecule to test for expression on the follicle cells. Future research should be aimed at determining what signals the follicle cell use in order to induce these receptors and what molecules are expressed on the surface of the apoptotic blebs.

Another issue to pursue is the fate of the follicle cells after they have performed their role as phagocytes. If one examines ovaries from 'starved' females, there is no backlog of degenerated egg chambers at the base of the ovary. They are removed entirely, including the surrounding follicle cells (see Figure 3b). This would suggest that once the follicle cells finish digesting the apoptotic blebs from the germline, they too undergo cell death. Expression of CIETDY in the follicle cells of starved females would allow the fate of these cells to be followed.

\section{Materials and Methods}

\section{Fly lines}

The UASp-diap1-15a and UASp-diap1-9-4 lines were obtained from Kim McCall (Boston, MA, USA). The UASp-p35 line was obtained from Andreas Bergmann (Houston, TX, USA). The GMR-Gal4, UAS-reaper, and nanos-Gal4VP16 lines were all obtained from Bloomington Stock Center. The p[COG-Gal4VP16; NGT40; nanos-Gal4VP16] fly line was created from three germline drivers. ${ }^{37}$ The P[CIETDY-3], nanosGal4VP16/TM3 flies were created by recombining P[CIETDY-3]/TM3 flies with the nanos-Gal4VP16 line.

\section{Antisera}

Purified polyclonal antibodies to GFP were purchased from Molecular Probes (Eugene, OR, USA) and Torrey Pines (La Jolla, CA, USA). Horseradish peroxidase-conjugated donkey anti-rabbit secondary antibody was purchased from Pierce Biotechnology, Inc. (Rockford, IL, USA). $1 \mathrm{~B} 1$ antibody to hu-li tai shao was obtained from Developmental Studies Hybridoma Bank (lowa City, IA, USA). Appropriate secondary antibodies conjugated to fluorophores were purchased from Molecular Probes (Eugene, OR, USA).

\section{Chemicals}

TO-PRO-1, YO-PRO-1, Alexa Fluor ${ }^{\circledR} 633$ phalloidin and rhodamine phalloidin were purchased from Molecular Probes (Eugene, OR, USA). 1,4 diazabizyclo[2.2.2]octane (DABCO) was purchased from ICN (Aurora, $\mathrm{OH}$, USA). Purified human caspase- 8 and human caspase- 3 were purchased from CHEMICON International, Inc. (Temecula, CA, USA). All other chemicals mentioned are from Sigma (St Louis, MO, USA).

\section{Creation of transgenes}

To create the CIETDY transgene, we used PCR primers (Invitrogen, Carlsbad, CA, USA) to amplify CFP and YFP and included nucleotides to create the linker sequence VIETDGA by a blunt ligation. Sense for YFP was 5'-GGGGATATCGAGACGGACGGGGCAATGGTGAGCAAGGGC GAGG-3' and antisense was 5'-CCCCAAGCTTGGTACCTTACTTGTA CAGCTCGTCCATGC-3'. Sense for CFP was 5'-GGGGATCCGGTACC ATGGTGAGCAAGGGCGAGG-3' and antisense was $5^{\prime}$-CCGGATCCGT TAACCTTGTACAGCTCGTCCATGC- $3^{\prime}$. These primers also contained restriction sites to ligate CFP and YFP into pBluescript $\mathrm{SK}^{-}$.

Primers were designed to amplify the putative mitochondrial outer membrane targeting sequence from CG9393. ${ }^{17}$ The sense primer $5^{\prime}$ CGGAGCGCAAGCTTCTAGACTCCGAGCTG-3' and the antisense primer 5'-CCCTCGAGGATCCGAATTCCGTCTAAGAGCGTGTCG-3' were used with cDNA made from wild-type RNA. This also created Hindlll and Xhol restriction sites and a stop codon to ligate it in-frame to the Cterminus of YFP. Once the entire construct was created and verified by sequencing, it was moved as a Kpnl/BamHI fragment into pUASp. ${ }^{25}$

To create the short-hairpin dronc RNAi transgene, we used the following primers to amplify the 265-1274 genomic piece of dronc and insert it as one side of a hairpin in UASp: ${ }^{25}$ sense $5^{\prime}$-GCGGTACCTGCT GATCAATGCACTGCGC- $3^{\prime}$ and antisense $5^{\prime}$-CGGGTACCGAGGAGGT CACCATTGTCAG- $3^{\prime}$. We then used the following primers: sense $5^{\prime}$ GGTCTAGACTGCTGATCAATGCACTGCGC-3' and antisense 5'-CCGC GGCCGCGAGGAGGTCACCATTGTCAG-3' to amplify the 265-765 CDNA piece of dronc and insert it in an inverted orientation into UASp ${ }^{25}$ so that it could make the other half of the dsRNA hairpin. The inclusion of an intron in the genomic piece allowed for easier cloning with traditional molecular biology techniques, but it was assumed to be processed out in the final mRNA transcript. ${ }^{38}$

\section{Creation of transgenic flies}

Drosophila $w^{1118}$ embryos were collected 20 min after egg laying, dechorionated by rolling on double-stick tape, and microinjected under mineral oil as described in Spradling ${ }^{39}$ with $0.65 \mathrm{mg} / \mathrm{ml}$ of UASp-CIETDY plasmid or UASp-dronc RNAi plasmid mixed with $0.4 \mathrm{mg} / \mathrm{ml}$ transposasecontaining plasmid RIO pActNPII. ${ }^{40} w^{+}$transformants were screened by eye color and crossed to $w^{1118} ; C y o / T(2 ; 3) a p^{X a}$ to balance and map. Homozygous stocks were maintained when possible.

\section{Fixation and preparation of ovaries for microscopy}

Ovaries were dissected into IMADS buffer, ${ }^{41}$ ovarioles were carefully 'combed' with forceps, and ovaries were fixed in 6:1 of heptane:devitelli-

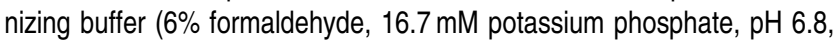
$75 \mathrm{mM}$ potassium chloride, $25 \mathrm{mM}$ sodium chloride, $3.3 \mathrm{mM}$ magnesium chloride) for $10 \mathrm{~min}$ at room temperature. ${ }^{42}$ After washing with $1 \times$ phosphate-buffered saline (PBS) and PBT $(0.5 \%$ bovine serum albumin, $0.3 \%$ Triton X-100, PBS), ovaries were treated with RNase A1 $(100 \mu \mathrm{g} / \mathrm{ml}$ in PBT), washed, and incubated with TO-PRO-1, YO-PRO-1, Alexa Fluor $^{\mathbb{R}} 633$ phalloidin and/or rhodamine phalloidin before storage in a DABCO/glycerol antifade solution at $4^{\circ} \mathrm{C}$. TO-PRO- 1 (1 pM in PBT) or Alexa Fluor ${ }^{\circledR} 633$ phalloidin $(1 \mathrm{U} / \mathrm{ml})$ was used whenever CIETDY was 
expressed. YO-PRO-1 (1 pM in PBT) and rhodamine phalloidin $(0.8 \mathrm{U} / \mathrm{ml}$ in PBT) were used simultaneously. Incubations with RNase and dyes were carried out for $1-2 \mathrm{~h}$ at room temperature or overnight at $4^{\circ} \mathrm{C}$. Before microscopy, the ovaries were mounted on microscope slides and a coverslip was sealed on with nail polish.

\section{Caspase cleavage and Western analysis}

Unfixed ovaries from 30 fattened P[CIETDY-2]; nanos-Gal4VP16 and P[CIETDY-3], nanos-Gal4VP16/TM3 females were placed into $0.3 \mathrm{ml}$ of buffer (25 mM sodium phosphate buffer, pH 7.0, $25 \mathrm{mM}$ sodium glutamate, $100 \mathrm{mM}$ sodium chloride, $2.5 \mathrm{mM}$ EDTA, $1 \mathrm{mM}$ DTT, $5 \mathrm{mM}$ benzamidine, $0.1 \mathrm{mM}$ PMSF, $1 \mu \mathrm{g} / \mathrm{ml}$ pepstatin A, $0.02 \%$ sodium azide). The ovaries were homogenized by hand and then sonicated on ice, and this extract was centrifuged at $14000 \times g$ to remove cell debris. The supernatant was reserved, and $35 \mu \mathrm{l}$ aliquots were incubated with various amounts of purified human caspase- $8(0,0.02,0.2$, and $2 \mathrm{U})$ for $2 \mathrm{~h}$ at $37^{\circ} \mathrm{C}$. Gel sample buffer was added, and the samples were boiled for $5 \mathrm{~min}$ to stop the reaction. Aliquots $(5 \mu \mathrm{l})$ of each sample were separated by $12 \%$ SDS-PAGE and transferred to nitrocellulose for Western analysis (http://www.westernblotting.org) with anti-GFP (diluted 1:10 000) to see cleavage. Primary and secondary antisera were diluted into PBS-T. ECL detection kit (Amersham, Piscataway, NJ, USA) was used to detect the signal from HRP-conjugated secondary antisera.

\section{Antibody staining of ovaries}

For immunofluorescence with $1 \mathrm{~B} 1$ antisera, fixed ovaries were incubated with 1B1 (1:10 dilution in PBT) at $4^{\circ} \mathrm{C}$ overnight. After washing with PBT, the ovaries with incubated with the appropriate species of secondary antibody (1:200 dilution in PBT) for 1-2 $\mathrm{h}$ at room temperature. Costaining for DNA or F-actin was performed simultaneously with this step. Then, ovaries were washed before storage in DABCO/glycerol antifade solution at $4^{\circ} \mathrm{C}$. Before microscopy, the ovaries were mounted on microscope slides and a coverslip was sealed on with nail polish.

\section{Microscopy and imaging}

Confocal micrographs were acquired with a Zeiss 510 META confocal using Zeiss LSM software, the gain was adjusted such that no CFP or YFP fluorescence was seen in the background, and .Ism images were converted into tif format using Graphic Converter (Lemke Software $\mathrm{GmbH}$, Peine, Germany). Images were histogram stretched in Photoshop 7.0 (Adobe, San Jose, CA, USA) before figures were created using Illustrator 10 (Adobe, San Jose, CA, USA). Unless exported from the Zeiss LSM software as TIFF files, images were false colored using Photoshop 7.0 in order to appear blue and yellow. In order to have images false-colored red, blue and yellow, Image $\mathrm{J}^{43}$ was used to merge the blue and yellow image with the red image.

\section{Acknowledgements}

We thank members of the Cooley Lab for their helpful advice and discussions. We also thank Andreas Bergmann for sharing a Drosophila line containing UASp-p35 ahead of publication, Nathalie Franc for sending Crq antisera, Bruce Hay for p35 antisera, Mike Buszczak for obtaining plasmids that contained CFP and YFP sequences, and the staff of the Center for Cell and Molecular Imaging at Yale University for confocal microscope services. SM was supported by the James Hudson Brown -
Alexander B. Coxe Postdoctoral Fellowship. LC is supported by NIH grant GM043301.

\section{References}

1. Baum JS, St George JP and McCall K (2005) Programmed cell death in the germline. Semin. Cell Dev. Biol. 16: 245-259.

2. Tilly JL (2001) Commuting the death sentence: how oocytes strive to survive. Nat. Rev. Mol. Cell Biol. 2: 838-848.

3. Cavaliere V, Taddei C and Gargiulo G (1998) Apoptosis of nurse cells at the late stages of oogenesis of Drosophila melanogaster. Dev. Genes Evol. 208: 106-112.

4. Foley K and Cooley L (1998) Apoptosis in late stage Drosophila nurse cells does not require genes within the H99 deficiency. Development 125: 1075-1082.

5. Nezis IP, Stravopodis DJ, Papassideri I, Robert-Nicoud M and Margaritis LH (2000) Stage-specific apoptotic patterns during Drosophila oogenesis. Eur. J. Cell Biol. 79: 610-620.

6. Nicholson DW and Thornberry NA (1997) Caspases: killer proteases. Trends Biochem. Sci. 22: 299-306.

7. Schwartz LM and Ashwell JD (eds) (2001) Apoptosis. Method Cell Biol. (San Diego, CA: Academic Press) Vol. 66.

8. Gumienny TL, Lambie E, Hartwieg E, Horvitz HR and Hengartner MO (1999) Genetic control of programmed cell death in the Caenorhabditis elegans hermaphrodite germline. Development 126: 1011-1022.

9. Mangahas PM and Zhou Z (2005) Clearance of apoptotic cells in Caenorhabditis elegans. Semin. Cell Dev. Biol. 16: 295-306.

10. Bangs $P$, Franc $N$ and White $K$ (2000) Molecular mechanisms of cell death and phagocytosis in Drosophila. Cell Death Differ. 7: 1027-1034.

11. McCall K and Steller H (1998) Requirement for DCP-1 caspase during Drosophila oogenesis. Science 279: 230-234.

12. Peterson JS, Barkett M and McCall K (2003) Stage-specific regulation of caspase activity in Drosophila oogenesis. Dev. Biol. 260: 113-123.

13. Leulier F, Rodriguez A, Khush RS, Abrams JM and Lemaitre B (2000) The Drosophila caspase Dredd is required to resist gram-negative bacterial infection. EMBO Rep. 1: 353-358.

14. Chew SK, Akdemir F, Chen P, Lu WJ, Mills K, Daish T, Kumar S, Rodriguez A and Abrams JM (2004) The apical caspase dronc governs programmed and unprogrammed cell death in Drosophila. Dev. Cell 7: 897-907.

15. Daish TJ, Mills K and Kumar S (2004) Drosophila caspase DRONC is required for specific developmental cell death pathways and stress-induced apoptosis. Dev. Cell 7: 909-915.

16. Xu D, Li Y, Arcaro M, Lackey M and Bergmann A (2005) The CARD-carrying caspase Dronc is essential for most, but not all, developmental cell death in Drosophila. Development 132: 2125-2134.

17. Drysdale RA, Crosby MA, Gelbart W, Campbell K, Emmert D, Matthews B, Russo S, Schroeder A, Smutniak F, Zhang P, Zhou P, Zytkovicz M, Ashburner M, de Grey A, Foulger R, Millburn G, Sutherland D, Yamada C, Kaufman T, Matthews K, DeAngelo A, Cook RK, Gilbert D, Goodman J, Grumbling G, Sheth $H$, Strelets V, Rubin G, Gibson M, Harris N, Lewis S, Misra S and Shu SQ (2005) FlyBase: genes and gene models. Nucleic Acids Res. 33: D390-D395.

18. Laundrie B, Peterson JS, Baum JS, Chang JC, Fileppo D, Thompson SR and McCall K (2003) Germline cell death is inhibited by P-element insertions disrupting the dcp-1/pita nested gene pair in Drosophila. Genetics 165: 1881-1888.

19. Drummond-Barbosa D and Spradling AC (2001) Stem cells and their progeny respond to nutritional changes during Drosophila oogenesis. Dev. Biol. 231: 265-278.

20. Buszczak M, Lu X, Segraves WA, Chang TY and Cooley L (2002) Mutations in the midway gene disrupt a Drosophila acyl coenzyme A: diacylglycerol acyltransferase. Genetics 160: 1511-1518.

21. Giorgi $F$ and Deri $P$ (1976) Cell death in ovarian chambers of Drosophila melanogaster. J. Embryol. Exp. Morphol. 35: 521-533.

22. Muro I, Monser K and Clem RJ (2004) Mechanism of Dronc activation in Drosophila cells. J. Cell Sci 117: 5035-5041. 
23. Di Fruscio M, Styhler S, Wikholm E, Boulanger MC, Lasko $P$ and Richard $S$ (2003) Kep1 interacts genetically with dredd/caspase-8, and kep1 mutants alter the balance of dredd isoforms. Proc. Natl. Acad. Sci. USA 100: 1814-1819.

24. Luo KQ, Yu VC, Pu Y and Chang DC (2001) Application of the fluorescence resonance energy transfer method for studying the dynamics of caspase-3 activation during UV-induced apoptosis in living HeLa cells. Biochem. Biophys. Res. Commun. 283: 1054-1060.

25. Rørth P (1998) Gal4 in the Drosophila female germline. Mech. Dev. 78: 113-118.

26. Cox RT and Spradling AC (2003) A Balbiani body and the fusome mediate mitochondrial inheritance during Drosophila oogenesis. Development 130: 1579-1590.

27. Werz C, Lee TV, Lee PL, Lackey M, Bolduc C, Stein DS and Bergmann A (2005) Mis-specified cells die by an active gene-directed process, and inhibition of this death results in cell fate transformation in Drosophila. Development 132: 5343-5352.

28. Chipuk JE and Green DR (2005) Opinion: do inducers of apoptosis trigger caspase-independent cell death? Nat. Rev. Mol. Cell Biol. 6: 268-275.

29. Sperandio S, de Belle I and Bredesen DE (2000) An alternative, nonapoptotic form of programmed cell death. Proc. Natl. Acad. Sci. USA 97: 14376-14381.

30. Degterev A, Huang Z, Boyce M, Li Y, Jagtap P, Mizushima N, Cuny GD, Mitchison TJ, Moskowitz MA and Yuan J (2005) Chemical inhibitor of nonapoptotic cell death with therapeutic potential for ischemic brain injury. Nat. Chem. Biol. 1: 112-119.

31. Edinger AL and Thompson CB (2004) Death by design: apoptosis, necrosis and autophagy. Curr. Opin. Cell Biol. 16: 663-669.

32. Rusten TE, Lindmo K, Juhasz G, Sass M, Seglen PO, Brech A and Stenmark H (2004) Programmed autophagy in the Drosophila fat body is induced by ecdysone through regulation of the PI3K pathway. Dev. Cell 7: 179-192.

33. Appelt U, Sheriff A, Gaipl US, Kalden JR, Voll RE and Herrmann M (2005) Viable, apoptotic and necrotic monocytes expose phosphatidylserine: cooperative binding of the ligand Annexin $\mathrm{V}$ to dying but not viable cells and implications for PS-dependent clearance. Cell Death Differ. 12: 194-196.

34. Didenko VV, Ngo H, Minchew CL, Boudreaux DJ, Widmayer MA and Baskin DS (2002) Caspase-3-dependent and -independent apoptosis in focal brain ischemia. Mol. Med. 8: 347-352.

35. Artal-Sanz M and Tavernarakis N (2005) Proteolytic mechanisms in necrotic cell death and neurodegeneration. FEBS Lett. 579: 3287-3296.

36. Monks J, Rosner D, Geske FJ, Lehman L, Hanson L, Neville MC and Fadok VA (2005) Epithelial cells as phagocytes: apoptotic epithelial cells are engulfed by mammary alveolar epithelial cells and repress inflammatory mediator release. Cell Death Differ. 12: 107-114.

37. Grieder NC, de Cuevas M and Spradling AC (2000) The fusome organizes the microtubule network during oocyte differentiation in Drosophila. Development 127: 4253-4264.

38. Piccin A, Salameh A, Benna C, Sandrelli F, Mazzotta G, Zordan M, Rosato E, Kyriacou CP and Costa R (2001) Efficient and heritable functional knock-out of an adult phenotype in Drosophila using a GAL4-driven hairpin RNA incorporating a heterologous spacer. Nucleic Acids Res. 29: E55.

39. Spradling A (1986) P element-mediated transformation In Drosophila: $A$ Practical Approach Roberts DB (ed) (Oxford, Washington DC: IRL Press), 175-197.

40. Beall EL, Mahoney MB and Rio DC (2002) Identification and analysis of a hyperactive mutant form of Drosophila P-element transposase. Genetics 162: 217-227.

41. Singleton K and Woodruff RI (1994) The osmolarity of adult Drosophila hemolymph and its effect on oocyte-nurse cell electrical polarity. Dev. Biol. 161: 154-167.

42. Cooley L, Verheyen E and Ayers K (1992) Chickadee encodes a profilin required for intercellular cytoplasm transport during Drosophila oogenesis. Cell 69: 173-184.

43. Rasband WS ( http://rsb.info.nih.gov/ij/, Bethesda, MD, 1997-2005).

Supplementary Information accompanies the paper on Cell Death and Differentiation website (http://www.nature.com/cdd) 